MATEC Web of Conferences 34, 01001 (2015)

DOI: $10.1051 /$ matecconf/ 20153401001

(C) Owned by the authors, published by EDP Sciences, 2015

\title{
Composition of silicon fibrous nanostructures synthesized using ultrafast laser pulses under ambient conditions
}

\author{
M. Sivakumar ${ }^{1,3, a}$, K. Venkatakrishnan ${ }^{2}$, B. $\operatorname{Tan}^{3}$ \\ Department of Sciences, Amrita School of Engineering, Amrita Vishwa Vidyapeetham, Amritanagar, Coimbatore, India 641112 \\ 2 Department of Mechanical and Industrial Engineering, Ryerson University, 350 Victoria Street, Toronto, Ontario, Canada, M5B 2 K3 \\ ${ }^{3}$ Department of Aerospace Engineering, Ryerson University, 350 Victoria Street, Toronto, Ontario, Canada, M5B 2K3
}

\begin{abstract}
In this study the composition of nanostructures generated owing to ablation of crystalline silicon using high repletion rate femtosecond laser under ambient condition is investigated. The web-like silicon fibrous nanostructures are formed in and around the laser irradiated area. Electron Microscopy investigation revealed that the nanostructures are made of nanoparticles of size about $40 \mathrm{~nm}$. In addition Micro-Raman analysis shows that the nanofibrous structures comprises a mixture of amorphous and polycrystalline silicon. X-ray photoelectron spectroscopy analysis reveals the oxidized and un-oxidized elemental states of silicon in the nanostructures. Moreover web-like fibrous nanostructures are generated due to condensation of super saturated vapour and subsequent nucleus growth in the laser induced plasma plume
\end{abstract}

\section{Introduction}

Recently semiconductor nanostructures have gained interest owing to their exceptional electronic properties. For instance silicon nanostructures find applications in areas like nano-electronics [1], photovoltaic devices [2], memory devices [3] and sensors [4]. Several methods have been investigated for the fabrication of silicon nanostructures such as slow combustion of silane [5], reduction of $\mathrm{SiCl}_{4}$ by $\mathrm{Na}$ [6], separation from porous $\mathrm{Si}$ [7], thermal evaporation [8], chemical vapour deposition [9], microwave discharge [10], RF sputtering [11], and high temperature aerosol techniques [12]. Studies have demonstrated that processing of materials using ultrafast laser pulses is a feasible route for the fabrication nanostructures with diverse materials [13]. Synthesis of crystalline silicon nanostructures using femtosecond laser pulses at $\mathrm{KHz}$ pulse repetition rate under vacuum in a gaseous environment is reported [14-17]. Recently a competent approach for increasing the rate of production and size reduction of silicon nanoparticles using femtosecond double-pulse laser ablation of silicon in ethanol is proposed [18]. Synthesis of silicon nanoparticles (Si NPs) by ablating porous silicon ( $\mathrm{pSi}$ ) in acetone using femtosecond laser pulses is reported [19]. With femtosecond laser pulse irradiation, materials reach extreme temperature and pressure in a very short time and also cool down rapidly. As a result states of materials which cannot be produced with laser pulses of extended duration are generated. Moreover processing of materials with femtosecond pulses results in minimal thermal and mechanical damage around the processed zone. This is

\footnotetext{
$\bar{a}$ Corresponding author: m_sivakumar@cb.amrita.edu
}

due to the fact that material transformation from solid to vapour / plasma prevents formation of liquid phase, and hence reduces effects due to thermal stress in the irradiated region. In recent times, investigations on irradiation of crystalline $\mathrm{Si}$ with ultrafast laser pulses at $\mathrm{MHz}$ pulse repetition rate in air shows formation of nanofibrous structures [20, 21]. By coupling vaporization of material by femtosecond laser pulses with controlled condensation from supersaturated vapour, size of the nanoparticles and formation of web-like structure can be controlled [22]. However for application point of view, it is essential to have a complete understanding of structural and compositional details of the fibrous nanostructures. In this context, the present study is aimed to investigate structural and chemical composition of silicon nanofibrous structures synthesized using $\mathrm{MHz}$ femtosecond laser pulses.

\section{Experimental methods}

The experimental setup used for this work is shown in Fig. 1. The laser source is a diode-pumped Yb-doped fiber oscillator/amplifier system $(1030 \mathrm{~nm})$ capable of producing maximum pulse energy up to $10 \mathrm{~mJ}$. The pulse repetition rate can be varied from $200 \mathrm{kHz}$ to $25 \mathrm{MHz}$. The sample used was crystalline silicon wafer with $<100>$ crystal orientation. The average power of laser was $7 \mathrm{~W}$ with a repetition rate of $4 \mathrm{MHz}$, pulse width $214 \mathrm{fs}$ and interaction time is $0.1 \mathrm{~ms}$. The laser beam is having Gaussian intensity profile and focused with $70 \mathrm{~mm}$ focal length lens. The focussed laser spot size is $10 \mu \mathrm{m}$ and estimated fluence was $2.3 \mathrm{~J} / \mathrm{cm}^{2}$. A computer 
controlled galvanometer system is used to irradiate the sample surface in air with an array of laser spots separated by a distance of $20 \mu \mathrm{m}$. Three samples were prepared with same laser parameters. First one was characterized using Raman spectroscopy followed by Scanning Electron Microscopy (SEM). The second and third samples were used for Transmission Electron Microscopy (TEM) and X-ray photoelectron spectroscopy (XPS) analysis. A JY Horriba LabRam system having frequency-doubled diode pumped $\mathrm{Nd}$ : YAG laser of $532 \mathrm{~nm}$ wavelength is used for MicroRaman investigations. The Raman scattered signal from sample surface is directed to spectrometer through confocal aperture. For TEM analysis, nanofibros structures were transferred to isopropanol solution by ultrasonification. Then a drop of solution in which nanostructures are dispersed was kept on copper grid and dried in air. XPS analysis was performed using a Thermo Scientific K-Alpha XPS spectrometer with AVANTAGE software for data acquisition and analysis. The samples were run at a take-off angle (relative to the surface) of $90^{\circ}$. A monochromatic Al K $\alpha$ X-ray source was used, with a spot area (on a $90^{\circ}$ sample) of $400 \mu \mathrm{m}^{2}$.

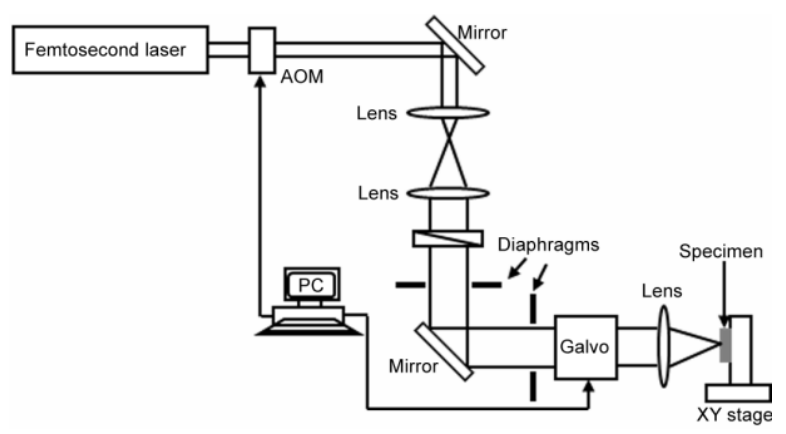

Figure 1. Experimental setup

\section{Results and discussion}

The nonlinear interaction of ultrafast laser pulses with silicon generates free electrons by Multiphoton and avalanche ionization processes. When number of electrons increases, absorption is dominated by Inverse Bremsstrahlung [23]. The electrons in quasi-equilibrium cool down by phonons emission at time of the order of $10^{-13}$ to $10^{-12} \mathrm{~s}$. Further equilibrium is reached by phonon relaxation due to their interaction with other phonon modes. The energy distribution at this stage is characterized by surface temperature of the sample. Moreover thermal equilibrium is reached within few picoseconds of laser energy deposition. After thermalization, the spatial energy distribution is characterized by temperature profile of the material. With femtosecond laser irradiation, this process is determined by optical absorption profile of silicon and hence steep temperature gradients could be produced. Thermal diffusion in the material takes place at time of the order of $10^{-11} \mathrm{~s}$. The entire process depends on optical and thermal properties of $\mathrm{Si}$. In addition with multipulse irradiation, absorption of incident radiation increases significantly after the first pulse. The heating rate of semiconductors irradiated with ultrafast laser pulses is of the order of $10^{14} \mathrm{~K} / \mathrm{s}$ [24]. Thus material is vaporized from the target into a plasma plume under ambient condition. Then condensation of saturated vapor begins by nucleation and subsequent growth of the nucleus is controlled by fast quenching. Hence nanoparticles are formed and redeposited on the surface of material. However for nanoparticles to fuse and form as fibrous nanostructures, a continuous source of vapour is needed to maintain the nucleus density at certain level. The required conditions for web-like fibrous nanostructure generation is provided by parameters which includes laser power, pulse repetition rate and laser interaction time with material. The basic nanoparticles formation mechanisms with irradiation of ultrafast laser pulses discussed are particles that are directly ejected from by expulsion of molten material from the irradiated area and/or particles formed by nucleation in the vapour of laser generated plasma $[25,26]$. In the present experiment, the irradiation of silicon surface with $\mathrm{MHz}$ repetition rate femtosecond laser pulses maintains a supersaturated vapour system against the atmospheric pressure. Consequently the non-equilibrium homogeneous nucleation process was initiated which brings the system to a new equilibrium state. In this process small liquid particles of silicon atoms are formed in the supersaturated vapour. While forming this new phase the energy of the system lowers and is proportional to volume of the new phase. The critical radius of nuclei formed through homogeneous nucleation and duration of particle growth depends on cooling rate of vapour plume and determines size of the nanoparticles. Since the samples are irradiated under ambient conditions, the cooling rate of vapour plume is very fast. As a result the size of the particles generated due to femtosecond laser ablation is very small. In addition the size and solidification time of nanoparticles determines self-assembly and porosity during redeposition as web-like fibrous nanostructures. In addition, relatively larger size nanoparticles are generated by expulsion of molten material from the irradiated area [26]. Either of these processes dominates depending on laser radiation fluence and pulse repetition rate. The fibrous nanostructures formed are piled up in and around the irradiated spot as shown in Fig. 2a. A higher magnification SEM image reveals individual strands of nanostructure, self-assembly and porosity (Fig. 2b). TEM image of nanoparticles that constitute the fibrous structure is depicted in Fig. 2c. The particles have not shown any partial coalescence with each other. Electron diffraction of nanoparticles shows that they are amorphous in nature.
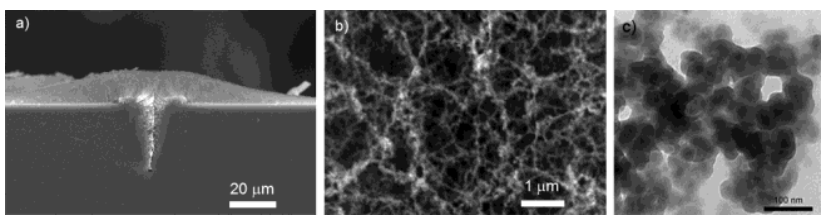

Figure 2 a) Cross-sectional SEM image of laser irradiated spot shows a pile of nanofibrous structures; b) Higher magnification image shows individual strands; c) TEM image of nanoparticles 
The presence of amorphous silicon as well as residual stresses in materials can be detected using Raman spectroscopy analysis [27]. The inelastic scattering of photons from laser radiation with lattice vibrations (phonons) of materials results Raman scattering. In the case of single crystals, phonons travel through lattice as wave and show dispersion which depend on their wavelength. In a polycrystalline material, the phonon propagation is interrupted at the grain boundary and confined within the grain. Nevertheless, from phonons point of view, a polycrystalline sample with micron grain size can be treated as a bulk crystal. In materials, when the grain size is smaller than 20 lattice parameters, consequences of phonon confinement are noticeable in the vibrational spectra. During laser irradiation of materials with fluence below melting threshold, phonons are excited due to a small part of energy. The excess energy outflows as radiation with slightly higher wavelength as compared to incident radiation. This difference in energy corresponds to vibrational energy of irradiated material. Consequently the frequency shift observed in Raman spectra of material reflects characteristics of bonds. Further the scattered frequencies reveal information about structure, defects and microstrain of the material. In crystalline solids, the incident photon momentum is negligibly small as conservation momentum selects phonons with zero momentum. Raman spectrum of crystalline silicon exhibits peak at $518.3 \mathrm{~cm}^{-1}$ (Fig. 3) due to optical phonons with zero momentum.

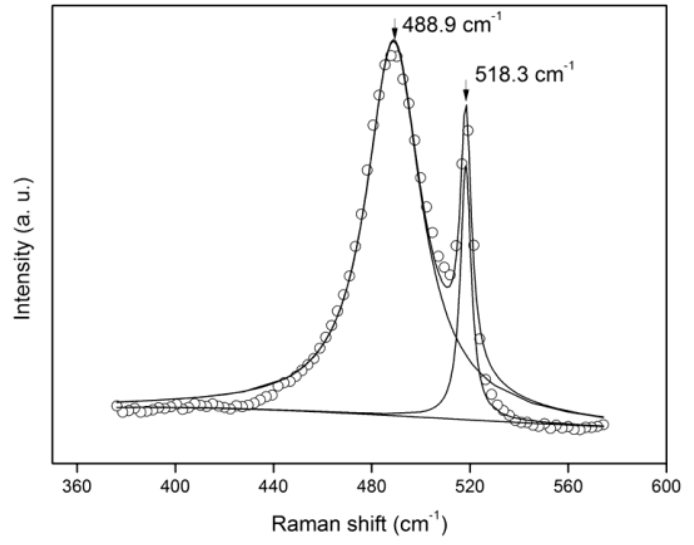

Figure 3. Micro-Raman spectrum of laser irradiated Si

In amorphous $\mathrm{Si}$, a broad Raman peak appears at $488.9 \mathrm{~cm}^{-1}$ due to relaxation of momentum selection rule. The FWHM of the crystalline and amorphous peaks are $5.4 \pm 0.6$ and $26.9 \pm 0.8 \mathrm{~cm}^{-1}$ respectively. The absorption coefficients and penetration depths of a-Si and c-Si for $532 \mathrm{~nm}$ wavelengths are $1.5 \times 10^{5} \mathrm{~cm}^{-1}, 67 \mathrm{~nm}$ and $1 \times 10^{4} \mathrm{~cm}^{-1}, 100 \mathrm{~nm}$ respectively [28]. The crosssectional SEM image (Fig. 2a) shows that the thickness of nanofibrous structure layer is around 20 microns. Consequently the peak in Raman spectrum appears at $518.3 \mathrm{~cm}^{-1}$ is mainly due to polycrystalline Si present in the layer. Moreover the layer consists of both amorphous and polycrystalline Si. From the intensity ratio, it can be concluded that there is significant contribution from a-Si as compared to $\mathrm{pc}-\mathrm{Si}$ in the nanofibrous structure. The surface elemental composition, electronic and chemical states of both laser irradiated and untreated samples were analyzed using XPS. The spectra are obtained by signals from surface / near surface layers of sample and hence would be able to distinguish between surface and substrate composition. In Fig. 4 the XPS survey spectra of unprocessed and laser irradiated samples are depicted. Both the surfaces are composed of silicon, oxygen, and carbon elements. Further Fig. 5 shows high resolution spectra of Si (2p) of both the samples. All the spectra were fitted with Gaussian functions to deconvolute component peaks for each type at its appropriate location and background was removed by Shirley subtraction method. In the Si 2 p spectra of both samples, two peaks corresponding to $\mathrm{SiO}_{2}$ and $\mathrm{Si}-\mathrm{Si}$ are observed [29]. The equivalent separations between these peaks are $\sim 3.7$ and $\sim 4.7 \mathrm{eV}$ respectively for unprocessed and laser irradiated samples.

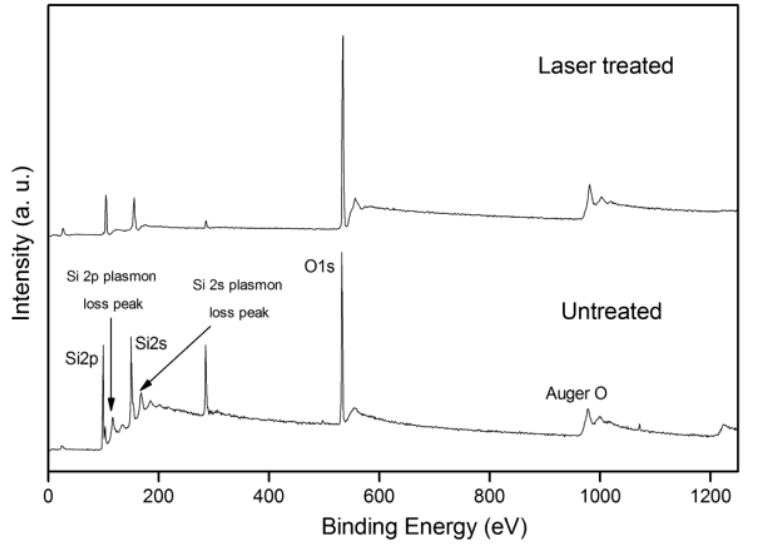

Figure 4. XPS survey spectra of laser treated and untreated Si

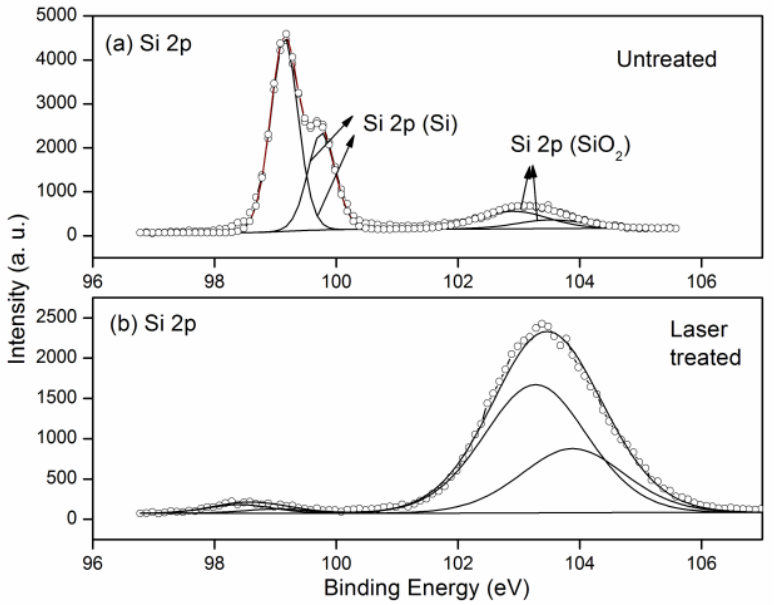

Figure 5. High resolution spectra of Si peaks of unprocessed and laser processed $\mathrm{Si}$

Further four possible oxidized and unoxidized states of silicon were identified. In the unprocessed sample the elemental Si states are specified by BE peaks at 99.2 and $99.8 \mathrm{eV}$ while oxidized silicon states are identified at 102.9 and $103.5 \mathrm{eV}$. The oxidized and unoxidized atomic concentrations are 81.6 and $18.4 \%$ respectively. On the other hand with laser irradiated surfaces, elemental peaks are at 99.3 and $100 \mathrm{eV}$ whereas oxidized peaks are identified at 104.3 and $104.9 \mathrm{eV}$. The corresponding atomic concentrations of elemental and oxidized silicon 
are 4.2 and $95.8 \%$ respectively. For unprocessed sample the signal from elemental unoxidized $\mathrm{Si}$ is much stronger than the oxidized Si peak (Fig. 5a). In other words, signal from silicon substrate is significant due to thin oxide layer. On the other hand, the oxide layer of nanofibrous structures has become thicker and accounts for a stronger signal from the oxidized state (Fig. 5b). Since XPS measurements analyze only the surface layer of few nanometres thick, the difference between spectra confirms the generation of oxidized fibrous nanofibrous structures with femtosecond laser irradiation of crystalline $\mathrm{Si}$ under ambient condition. The analysis shows that, nanoparticles were oxidized during nucleation process. This is due to the fact that Si species in the high temperature plasma plume possess greater affinity towards oxygen present in the atmosphere. In addition the oxygen atoms and ions could also diffuse into nanoparticles during the process. The complete oxidation state of nanostructures is more stable as compared to intermediate oxidation states.

\section{Conclusions}

The constituents and chemical composition Si fibrous nanostructures synthesized by irradiation of crystalline silicon with $\mathrm{MHz}$ repetition rate femtosecond laser pulses under ambient condition is investigated. Electron microscopy investigation reveals that the fibrous nanostructures are observed inside and around the laser irradiated area. Moreover the strand of nanostructures is comprises of amorphous and polycrystalline silicon nanoparticles. The results are further evidenced by Micro- Raman spectroscopy analysis. The Raman spectrum contains peaks that corresponds to amorphous and polycrystalline silicon. The chemical analysis revealed the higher proportion of oxidized silicon states of nanostructures as compared to elemental silicon states.

\section{References}

1. S. Tiwari, F. Rana, H. Hanafi, A. Hartstein, E.F. Crabbe, K. Chan, Appl. Phys. Lett. 68 (1996)

2. S. Prezioso, S.M. Hossain, A. Anopchenko, L. Pavesi, M. Wang, G. Pucker, P. Bellutti, Appl. Phys. Lett. 94 (2009)

3. T.Z. Lu, M. Alexe, R. Scholz, V. Talalaev, R.J. Zhang, M. Zacharias, J. Appl. Phys. 100 (2006)

4. P. Alpuim, S.A. Filonovich, C.M. Costa, P.F. Rocha, M.I. Vasilevskiy, S. Lanceros-Mendez, C. Frias, A.T. Marques, R. Soares, C. Costa, J. Non-Cryst. Solids, 354 (2008)

5. A. Fojtik, H. Weller, S. Fiechter, A. Henglein, Chem. Phys. Lett., 134 (1987)

6. J. Zou, P. Sanelle, K.A. Pettigrew, S.M. Kauzlarich, J. Cluster Sci., 17 (2006)

7. R.A. Bley, S.M. Kauzlarich, J.E. Davis, H.W.H. Lee, Chem. Mater., 8 (1996)

8. Y. Qin, X.N. Zhang, K. Zheng, H. Li, X.D. Han, Z. Zhang, Appl. Phys. Lett. 93 (2008)
9. D.J. DiMaria, J.R. Kirtley, E.J. Pakulis, D.W. Dong, T.S. Kuan, F.L. Pesavento, T.N. Theis, J.A. Cutro, S.D. Brorson, J. Appl. Phys. 56 (1984)

10. H. Takagi, H. Ogawa, Y. Yamazaki, A. Ishizaki, T. Nakagiri, Appl. Phys. Lett. 56 (1990)

11. Y. Zhu, H. Wang, P.P. Ong, Appl. Surf. Sci. 171 (2001)

12. J. Holm, J.T. Roberts, J. Am. Chem. Soc., 129 (2007)

13. S. Eliezer, N. Eliaz, E. Grossman, D. Fisher, I. Gouzman, Z. Henis, S. Pecker, Y. Horovitz, M. Fraenkel, S. Maman, Y. Lereah, Physical Review B, 69 (2004)

14. B.R. Tull, J.E. Carey, E. Mazur, J.P. McDonald, S.M. Yalisove, Mrs Bulletin, 31 (2006)

15. B.R. Tull, J.E. Carey, M.A. Sheehy, C. Friend, E. Mazur, Appl. Phys. A-Mater. 83 (2006)

16. M.Y. Shen, C.H. Crouch, J.E. Carey, E. Mazur, Appl. Phys. Lett. 85 (2004)

17. M.A. Sheehy, L. Winston, J.E. Carey, C.M. Friend, E. Mazur, Chem. Mater., 17 (2005)

18. X. Li, G. Zhang, L. Jiang, X. Shi, K. Zhang, W. Rong, J. Duan, Y. Lu, Opt. Express. 23 (2015)

19. V.S. Vendamani, S. Hamad, V. Saikiran, A.P. Pathak, S. Venugopal Rao, V.V. Ravi Kanth Kumar, S.V.S. Nageswara Rao, J. Mater. Sci. 50 (2014)

20. B. Tan, K. Venkatakrishnan, Opt. Express. 17 (2009)

21. A.S. Mahmood, M. Sivakumar, K. Venkatakrishnan, B. Tan, Appl. Phys. Lett. 95 (2009)

22. S.T. Li, S.J. Silvers, M.S. ElShall, J. Phys. Chem. B, 101 (1997)

23. J. Kruger, W. Kautek, Laser Phys. 9 (1999)

24. D. vonderLinde, K. SokolowskiTinten, J. Bialkowski, Appl. Surf. Sci. 109 (1997)

25. T.E. Itina, T.E. Itina, N. Bouflous, N. Bouflous, J. Hermann, J. Hermann, P. Delaporte, P. Delaporte, Proc. SPIE, 8414 (1899)

26. M. Sivakumar, K. Venkatakrishnan, B. Tan, Nanotechnology, 21 (2010)

27. A.K. Arora, M. Rajalakshmi, T.R. Ravindran, Encyclopedia of Nanoscience and Nanotechnology 8 (2004)

28. A. Matsuno, E. Takii, T. Eto, K.-i. Kurobe, K. Shibahara, Nucl. Instrum. Methods Phys. Res., Sect. B 237 (2005)

29. W.K. Choi, F.W. Poon, F.C. Loh, K.L. Tan, J. Appl. Phys. 81 (1997) 